\title{
Stability of trapped Bose-Einstein condensates
}

\author{
F. Kh. Abdullaev, ${ }^{1, *}$ A. Gammal, ${ }^{1}$ Lauro Tomio, ${ }^{1}$ and T. Frederico ${ }^{2}$ \\ ${ }^{1}$ Instituto de Física Teórica, Universidade Estadual Paulista, 01405-900 São Paulo, Brazil \\ ${ }^{2}$ Departamento de Física, Instituto Tecnológico da Aeronáutica, Centro Técnico Aeroespacial, 12228-900 São José dos Campos, \\ São Paulo, Brazil
}

(Received 18 August 2000; revised manuscript received 27 October 2000; published 12 March 2001)

\begin{abstract}
In three-dimensional trapped Bose-Einstein condensate (BEC), described by the time-dependent GrossPitaevskii-Ginzburg equation, we study the effect of initial conditions on stability using a Gaussian variational approach and exact numerical simulations. We also discuss the validity of the criterion for stability suggested by Vakhitov and Kolokolov. The maximum initial chirp (initial focusing defocusing of cloud) that can lead a stable condensate to collapse even before the number of atoms reaches its critical limit is obtained for several specific cases. When we consider two- and three-body nonlinear terms, with negative cubic and positive quintic terms, we have the conditions for the existence of two phases in the condensate. In this case, the magnitude of the oscillations between the two phases are studied considering sufficient large initial chirps. The occurrence of collapse in a BEC with repulsive two-body interaction is also shown to be possible.
\end{abstract}

DOI: 10.1103/PhysRevA.63.043604 PACS number(s): 03.75.Fi, 32.80.Pj, 42.50.Md, 03.75.-b

\section{INTRODUCTION}

In trapped Bose-Einstein condensates (BEC's), it is well known that collapse occurs when the two-body interaction is attractive and if the number of atoms $N$ exceeds a critical value $N_{c}$, as in the case of atomic condensates with ${ }^{7} \mathrm{Li}[1]$. In this case, experiments with attractive two-body interaction have been performed [2], with results consistent with the limitation in the number of atoms and with the growth and collapse scenario. As the nonlinear terms presented in the nonlinear Schrödinger equation (NLSE) (cubic, quintic, and so on) are due to the first terms of an expansion of the effective many-body interaction in the mean-field approximation [3], a study of stability of the equation that describes BEC's is necessary, considering the observed few-body interactions that occur in the atomic gas. When the overall sign of the effective many-body interaction is negative, we have the conditions for the occurrence of collapse, with the stability of the condensate being restricted by the number of atoms.

We should stress that the criteria for stability usually involves one parameter: the number of atoms. Here, in order to introduce a criterion for stability of a trapped condensate with two- and three-body effective interactions, we introduce another parameter: a chirp, that is related to the initial focusing (defocusing) of the cloud. Without a trap, a chirp parameter was previously introduced in Ref. [4] to study the stability of the NLSE; with a trap, the stability was studied in Refs. [5-7]. One should also note that an oscillating condensate always has a chirped wave function, where the chirp is proportional to the time variation of the width, which is a periodic function of time. Thus a real condensate, for example in optical traps, always has a nonzero initial chirp, that should be taken into account.

Collapses for two- and three-dimensional geometries of a

\footnotetext{
*On leave of absence from Physical-Technical Institute, Tashkent, Uzbekistan.
}

NLSE with trap potential were investigated in Refs. [1,8], where the corresponding critical numbers were obtained $[9,10]$. These works are extensions of Zakharov and coworkers' theory of collapse [11-14] to the case when an additional trap potential is included. The trap potential helps to prevent the collapsing process, which cannot be avoided if the number of atoms exceeds the critical limit. In Refs. $[8,15]$, considering the moments method in the analysis of the equation for the average squared value of the width, $\left\langle a^{2}\right\rangle$, the influence of the corresponding initial condition on the collapse was emphasized.

Variational approaches were also used by many authors for an analysis of the stability of NLSE, and proved to be useful in many aspects (see Ref. [4], for example). The variational approach (VA), using a simple Gaussian ansatz, gives a reasonable description of the conditions for collapse, and also an approximate value of the critical number of atoms. It is interesting to apply the time-dependent variational approach to describe the dynamics of a BEC with attractive two-body interactions, and in particular to study the influence of the initial conditions (using a chirp constant $b$ in the wave function) on the stability of a BEC. This can be important for condensation in optical traps and in strongly inhomogeneous traps. Also, recently (in Ref. [16]) a stability analysis was performed, using both VA and exact calculations, considering that the condensate can unstabilize by tunneling, due to quantum fluctuations.

When the two-body interaction term is switched off, for example by tuning an external magnetic field [17], the threebody interaction term can play an important role. Thus it is interesting to study collapse conditions in a model with a trap potential term and an attractive three-body interaction (quintic) term. Recently, in Ref. [18], the collapse in a onedimensional (1D) model with attractive three-body interactions was investigated. The trap was narrow, and induced a radiative loss of atoms, reducing the number below the critical value, which made an arrest of the collapse possible. The two-dimensional case, with cubic nonlinearity (two-body interactions), may be useful in modulation theory around the 
Townes soliton [19]. Then it is possible to estimate the role played by the continuum.

In the present work, we first consider a review of the stability criterions, without application of initial chirp. Next we analyze the role of an initial chirp in the wave function of a stable condensate with an attractive two-body nonlinear term, which can be useful for actual experimental analysis in BEC's. A generalization of this study of stability is considered with the inclusion of a three-body interaction term in the NLSE. Such a study can be relevant to the perspective of atomic systems with enhanced three-body effects, that can occur as the two-body scattering length is altered [17].

When a positive three-body interaction term is included in a trapped 3D NLSE with negative two-body interaction, there already exists the possibility of extending the region of stability of the equation to a larger number of atoms, with the occurrence of two stable phases in the condensate [20,21]. In Ref. [3], the frequency of the collective excitations in denser (liquid) and dilute (gas) phases of the condensate was calculated. Here we investigate a bifurcation phenomenon related to the time variation of the width parameter of the theory, by switching the oscillations from one phase to the other. The present study is done with the aid of a time-dependent variational approach for the collapse of the atomic cloud in a 3D model with harmonic trap potential. We compare the predictions of the VA with exact numerical simulations of the partial differential equation (PDE). As detailed in our conclusions, the Gaussian VA gives a good estimate of the observables in the region of stability, and begins to deviate from exact results when the system is close to the collapse conditions, and particularly for the unstable solutions (maxima) of the total energy. For this regions, where the Gaussian VA fails, one should improve the ansatz or take the results as a qualitative picture to guide the exact numerical calculations.

In cases without traps, the NLSE was previously studied by Vakhitov and Kolokolov (VK) [22], where a criterion for stability was settled. This and other criteria for stability in nonlinear systems have been studied and extensively used by many authors (see Refs [23,12], and references therein). The VK criterion was recently detailed in Ref. [12]. When a trap potential was added to a NLSE with a negative cubic term, an alternative stability criterion was derived in Ref. [7], after considering similarities between BEC atomic systems and compact objects like neutron stars. Recently, the VK criterion for a trapped BEC with a cubic term was formally demonstrated in Ref. [24].

In recent numerical studies $[3,21,20]$ one can also observe that the VK criterion is not generally valid; it cannot be extended, for example, to the case of a trapped system with both attractive cubic and repulsive quintic terms. The solutions can be stable irrespective of the sign of the derivative of the eigenvalues of the NLSE with respect to the number of atoms, $d \mu / d N$. When $g_{3}$ is positive, and for a large enough number of atoms, the NLSE is stable and $d \mu / d N$ can be positive (see, for example, Ref. [3]), implying that the criterion cannot be extended to a trapped NLSE with two- and three-body terms. Here the nonvalidity of the VK criterion is clearly verified when we have a positive harmonic trap, with two- and three-body nonlinear terms with opposite signs.

We extend the study of the stability of the NLSE with cubic (two-body) and quintic (three-body) terms, through the analysis of chirp response, and through the frequency of collective excitations. By applying the perturbation techniques to the theory of nonlinear oscillations [25], we also derive the frequency of weakly nonlinear collective excitations. This study extends the numerical calculations performed in Ref. [3] for the frequencies of collective excitations.

One should also note that such a study is of actual interest, as recently Ref. [26] reported observations of nonlinear oscillations in BEC's of a gas with rubidium atoms. The present study can be of interest not only to atomic BEC's but also to other branches of physics and mathematics, where nonlinear effective interactions are added to a trapped potential in the time-dependent Schrödinger equation, in, e.g., optics and soliton physics.

In Sec. II, we give a description of the model, using a variational approach for a system with $D$ dimensions. In Sec. III, we derive an expression for the chirp parameter that is considered in our analysis of stability. The maximum initial chirp to keep the system oscillating in the same phase, around a minimum of the energy, without collapsing or without a phase transition, is obtained in this section by considering both variational and exact numerical calculations. In Sec. IV, we follow the study of Sec. III, and obtain frequencies for the linear and nonlinear oscillations. In this section we consider the presence of both cubic and quintic terms, in several different configurations. The VK criterion of stability is discussed in Sec. V. Finally, in Sec. VI, we present our concluding remarks.

\section{DESCRIPTION OF THE MODEL}

In this section we will consider the dynamics described by a NLSE with a harmonic potential and with cubic and quintic terms. Using a variational approach for a system with $D$ dimensions, from the Lagrangian we derive an expression for the anharmonic potential considering the mechanical analogy.

The time-dependent Gross-Pitaevskii-Ginzburg equation, that will be considered in the present approach, is given by

$$
2 \mathrm{i} \psi_{t}=-\Delta \psi+V \psi+\lambda_{2}|\psi|^{2} \psi+\lambda_{3}|\psi|^{4} \psi .
$$

In this equation and in the following equations, the explicit space and time dependences of the variables and parameters are implicit, unless it is necessary or convenient for clarity. The time derivatives will be denoted by indices $t$. In Eq. (1) we assume dimensionless variables: the unit of energy is $\hbar \omega / 2$, the unit of length is $\sqrt{\hbar /(m \omega)}$, and the unit of time is $1 / \omega . V \equiv V(\vec{r})$ is a static trap potential, and we assume a harmonic oscillator with spherical symmetry given by $V$ $=r^{2}$ in the present units. $\lambda_{2}$ and $\lambda_{3}$ are the parameters of the two- and three-body interactions, which in general can be complex quantities. The imaginary parts of $\lambda_{2}$ and $\lambda_{3}$ describe, the effects of inelastic two- and three-body collisions on the dynamics of BEC's, respectively. In the present paper, we are not considering dissipative terms, and such cubic and 
quintic parameters are real. In order to compare with the formalism given in Ref. [3], $\lambda_{2}$ is proportional to the twobody scattering length $a_{s c}$, and is given by $\lambda_{2} \equiv 8 \pi a_{s c}$.

The chemical potential $\mu$, is given by the eigenvalue solutions of Eq. (1), with $\psi(r, t)=e^{-\mathrm{i}(\mu t / 2)} \varphi(r)$ :

$$
\mu \varphi=-\Delta \varphi+r^{2} \varphi+\lambda_{2}|\varphi|^{2} \varphi+\lambda_{3}|\varphi|^{4} \varphi .
$$

The Lagrangian density corresponding to Eq. (1) is given by

$$
\mathcal{L}=2 \operatorname{Im}\left(\psi_{t}^{*} \psi\right)-|\nabla \psi|^{2}-r^{2}|\psi|^{2}-\frac{\lambda_{2}}{2}|\psi|^{4}-\frac{\lambda_{3}}{3}|\psi|^{6} .
$$

\section{A. Gaussian variational approach}

To analyze the dynamics of BEC's under two- and threebody interactions, it is convenient to follow the variational approach developed in Refs. [27,18]. This approach was successfully employed, recently, in Ref. [28] for a BEC with two-body interaction. We choose the simple Gaussian ansatz

$$
\psi(r, t)=A(t) \exp \left(-\frac{r^{2}}{2 a^{2}(t)}+\mathrm{i} \frac{b(t) r^{2}}{2}+\mathrm{i} \phi(t)\right),
$$

where $A(t)$ is the amplitude, $a(t)$ is the width, $\phi(t)$ is the linear phase of the condensate, and $b(t)$ is the "chirp" parameter previously discussed.

Without dissipative terms, the normalization of the wave function is conserved, and given by the number of particles $N$. The mean-square radius and the normalization, for a system with $D$ dimensions, are

$$
\left\langle r^{2}\right\rangle=\frac{D}{2} a^{2}, \quad \text { and } \quad N=A^{2}(\sqrt{\pi} a)^{D}=\text { const. }
$$

For a system with $D$ dimensions and radial symmetry, the averaged Lagrangian expression is given by

$$
\begin{aligned}
-L= & (\sqrt{\pi} a)^{D} A^{2}\left[2 \phi_{t}+\frac{D}{2} a^{2}\left(b_{t}+\frac{1}{a^{4}}+b^{2}+1\right)\right. \\
& \left.+\frac{\lambda_{2} A^{2}}{2 \sqrt{2}^{D}}+\frac{\lambda_{3} A^{4}}{3 \sqrt{3}^{D}}\right],
\end{aligned}
$$

where

$$
\delta \int L d t=0 \quad \text { where } \quad L=\int \mathcal{L}(r, t) d \vec{r} .
$$

Analyzing the corresponding Euler-Lagrange equations,

$$
\frac{\partial L}{\partial \eta_{i}}-\frac{d}{d t} \frac{\partial L}{\partial \dot{\eta}_{i}}=0
$$

where $\eta_{i}$ refer to the variational parameters $[A(t), a(t), b(t)$, and $\phi(t)]$, we obtain

$$
a_{t}=a b
$$

and

$$
b_{t}=\frac{1}{a^{4}}-b^{2}-1+\frac{\lambda_{2} N a^{-(D+2)}}{2(2 \pi)^{(D / 2)}}+\frac{2 \lambda_{3} N^{2} a^{-(2 D+2)}}{3(\pi \sqrt{3})^{D}} .
$$

Equation (9) expresses the chirp parameter $b$ via initial focusing (defocusing) $\left(a_{t}\right)$ of the wave function. Combining Eqs. (9) and (10), we have

$$
\begin{gathered}
a_{t t}=\frac{1}{a^{3}}-a+\frac{P}{a^{(D+1)}}+\frac{Q}{a^{(2 D+1)}}, \\
P \equiv \frac{\lambda_{2} N}{2 \sqrt{(2 \pi)^{D}}}, \\
Q \equiv \frac{2 \lambda_{3} N^{2}}{3(\pi \sqrt{3})^{D}}=\frac{(2)^{(D+3)}}{(3)^{(D / 2+1)}} \frac{\lambda_{3}}{\lambda_{2}^{2}} P^{2} .
\end{gathered}
$$

Comparing the result of $a_{t t}$ with $D=2$, from Eq. (11), with that of Ref. [28], we observe that the resulting equation obtained by the variational approach coincides with one obtained by the moments method [29]. The equation is also close to the one obtained by the modulation method for the 2D Townes soliton [19]. When $D=3$, expressing the above definitions by the parameters given in Ref. [21], where $\lambda_{2}$ $=8 \pi a_{s c}$ in our dimensionless units ( $a_{s c}$ is the two-body scattering length), and $N=n /\left(2 \sqrt{2}\left|a_{s c}\right|\right)$,

$$
|P|=\frac{n}{2 \sqrt{\pi}} \quad \text { and } \quad Q=\frac{8 n^{2}}{9 \pi \sqrt{3}} g_{3} .
$$

The anharmonic potential is derived using the mechanical analogy. By explicitly using the dimensions in Eq. (5),

$$
\begin{gathered}
R \equiv \sqrt{\left\langle r^{2}\right\rangle}=\sqrt{\frac{D}{2}} a\left(\frac{\hbar}{m \omega}\right)^{1 / 2}, \\
m \frac{d^{2}}{d t^{2}} R=\sqrt{\frac{D}{2}} a_{t t}\left[m \omega^{2}\left(\frac{\hbar}{m \omega}\right)^{1 / 2}\right],
\end{gathered}
$$

results that can be identified with

$$
-\frac{\partial U_{P}(R)}{\partial R}=-\left(\frac{\hbar \omega}{2}\right) \sqrt{\frac{2}{D}}\left(\frac{m \omega}{\hbar}\right)^{1 / 2} \frac{\partial U}{\partial a},
$$

where

$$
U_{P}(R) \equiv U(a) \frac{\hbar \omega}{2}
$$

The total energy for a BEC system with $N$ particles is given by

$$
E_{T}=N U_{P}(R)=N U(a) \frac{\hbar \omega}{2} \equiv E(a) \frac{\hbar \omega}{2} .
$$

So, for the dimensionless quantities, we obtain 


$$
\frac{\partial U}{\partial a}=-(D) a_{t t}
$$

With Eq. (11), we have the anharmonic potential in dimensionless units:

$$
U(a)=\frac{D}{2}\left(a^{2}+\frac{1}{a^{2}}\right)+\frac{P}{a^{D}}+\frac{Q}{2 a^{(2 D)}} .
$$

Correspondingly, from Eq. (2), the chemical potential is given by

$$
\mu(a)=\frac{D}{2}\left(a^{2}+\frac{1}{a^{2}}\right)+\frac{2 P}{a^{D}}+\frac{3 Q}{2 a^{(2 D)}} .
$$

The asymptotic limit $a \rightarrow \infty$ for $U(a)$ is the same as for the oscillator $[U(\infty) \rightarrow \infty]$, and is not determined by the parameters $P$ and $Q$. So, the only limiting condition that is strongly affected by these parameters is the limit $a=0$. When $Q \neq 0$, the three-body term is dominant in the limit $U(0) \approx Q /\left(2 a^{2 D}\right)$. Two cases have to be considered: $Q<0$ gives us $U(0) \rightarrow-\infty$, and $Q>0$ will give us $U(0) \rightarrow+\infty$. As the other end is fixed by the oscillator condition $[U(\infty)$ $\rightarrow+\infty]$, in the first case $(Q<0)$ we cannot obtain more than one maximum and one minimum for finite $a$, that will depend on the sign and relative value of the parameter $P$. The case $Q>0$ can be very interesting if $P<0$ : at both ends $U(a)$ goes to $+\infty$, such that in between we can have two minima and one maximum for the total energy. The two minima will represent two possible phases, and a rich dynamics can be described by the NLSE. From this analysis, we should note that a phase transition is possible only when $Q>0$, with $P<0$. When $Q \leqslant 0$, there is only a single phase.

\section{B. Critical parameters for stability}

The relevant extremes of $U(a)$ are given by the real and positive roots of

$$
a_{s}^{2}-\frac{1}{a_{s}^{2}}-\frac{P}{a_{s}^{D}}-\frac{Q}{a_{s}^{2 D}}=0 .
$$

In such extreme positions, we obtain

$$
\begin{aligned}
& U\left(a_{s}\right)=D a_{s}^{2}+\frac{P}{2 a_{s}^{D}}(2-D)+\frac{Q}{2 a_{s}^{2 D}}(1-D), \\
& \mu\left(a_{s}\right)=D a_{s}^{2}+\frac{P}{2 a_{s}^{D}}(4-D)+\frac{Q}{2 a_{s}^{2 D}}(3-D) .
\end{aligned}
$$

In Table I we show the particular cases, from the above three equations, where exact analytical solutions can be found for the extremes of $U$ and the corresponding critical parameters for stability. We note that, once a solution for a system with $D$ dimensions and $P=0$ is known, the same solution can be used for the roots of a system with $2 D$ dimensions and $Q=0$, by just exchanging $P$ and $Q$. When $D$
TABLE I. For lower dimensions, we show the results of particular cases where exact analytical solutions can be found. $a_{s}$ 's are real and positive roots of the mean-square radius that extremize the total energy $\left[E_{T}=N U(\hbar \omega / 2)\right] ; P_{c}$ and $Q_{c}$ are the critical parameters for stability of the NLSE in just one local minimum. For $D$ $=3$, when $P$ and $Q$ are nonzero, we show just one critical set of parameters for the existence of two phases. $U_{c}$ and $\mu_{c}$ are the corresponding critical values of $U(a)$ and the chemical potential $\mu(a)$.

\begin{tabular}{ccccccc}
\hline \hline$D$ & $(P, Q)$ & $\left(P_{c}, Q_{c}\right)$ & $a_{s}^{4}$ & $a_{c}^{4}$ & $U_{c}$ & $\mu_{c}$ \\
\hline 1 & $(0, Q)$ & $(0,-1)$ & $1+Q$ & 0 & 0 & $\frac{-1}{a_{c}^{2}}$ \\
2 & $(P, 0)$ & $(-1,0)$ & $1+P$ & 0 & 0 & $\frac{-1}{a_{c}^{2}}$ \\
3 & $(0, Q)$ & $\left(0, \frac{2 \sqrt{3}}{9}\right)$ & Eq. $(22)$ & $\frac{4}{3}$ & $\frac{5 \sqrt{3}}{4}$ & $\frac{17 \sqrt{3}}{12}$ \\
3 & $\left(P, P^{8}\right)$ & $\left(-\left(\frac{1}{8}\right)^{1 / 4}, \frac{1}{64}\right)$ & Eq. $(22)$ & $\frac{1}{8}$ & $\frac{3 \sqrt{2}}{2}$ & $\frac{-\sqrt{2}}{4}$ \\
4 & $(P, 0)$ & $\left(\frac{2 \sqrt{3}}{9}, 0\right)$ & Eq. $(22)$ & $\frac{4}{3}$ & $\frac{5 \sqrt{3}}{2}$ & $\frac{8 \sqrt{3}}{3}$ \\
6 & $(P, 0)$ & $\left(\frac{-1}{4}, 0\right)$ & $\frac{1}{2} \pm \sqrt{\frac{1}{4}+P}$ & $\frac{1}{2}$ & $\frac{3 \sqrt{2}}{4}$ & $\frac{7 \sqrt{2}}{2}$ \\
\hline \hline
\end{tabular}

$=2$, the result $P_{c}=-1$ for the particular case when the quintic term is zero $(Q=0)$ is well known, and corresponds to $\nu=2$ in Ref. [30]. The solutions will be unstable for $P=$ $-|P|$ with $|P|>1$.

Actually, one of the interesting cases occurs when the cubic term is zero $(P=0)$, for $D=1$ and 3 , in view of the possibility of altering the two-body scattering length by an external magnetic field [17]. If this condition is realized ( $P$ $=0$ ), the effect of the three-body nonlinear term in the mean-field approximation of the condensate will be enhanced. In case such a three-body (quintic) term is negative, as shown in Table I, the present variational ansatz gives analytical estimates for the critical parameters, and for physical quantities such as the mean-square-radius, energy and chemical potentials. In Sec. IV we study this case in more detail.

Other particular cases occur when phase transitions are possible. As explained above, these situations can only occur if both parameters $P$ and $Q$ are nonzero and $Q>0$ with $P$ $<0$. An absolute collapse is not possible in the condensate; hower, one can obtain transitions between two phases. This implies that, for a system with a two-body attractive interaction and a three-body repulsive one, as we increase the number of atoms in the condensate $(|P|$ increases), we can reach a critical limit where only one phase (stable) remains. In other words, for a particular value of $P=P_{c}$, the position $a$ of one of the minima of $U$ coincides with the maximum, and we have an inflection point of $U$. 


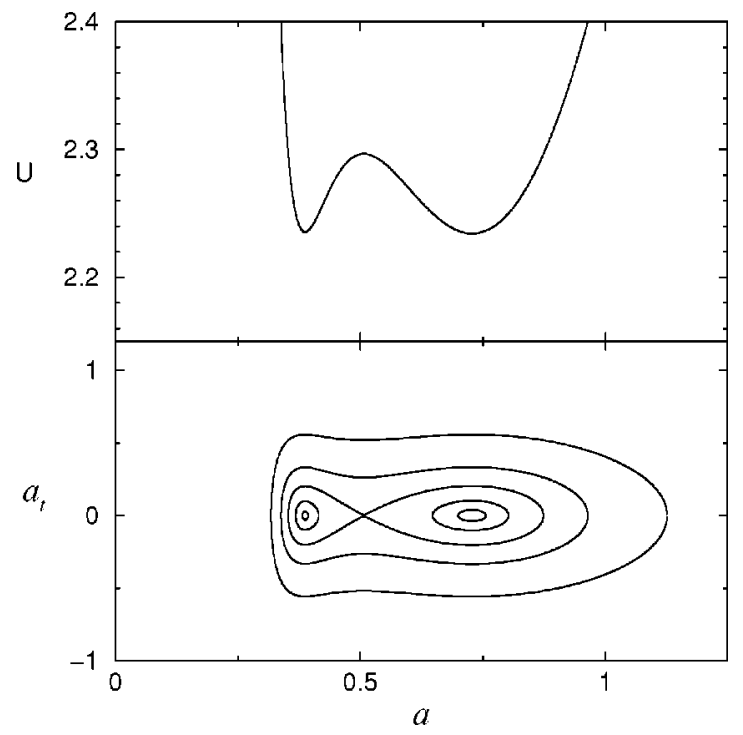

FIG. 1. For a stable BEC with $\lambda_{2}<0, \lambda_{3}>0$, and $D=3$, we show a variational plot of the potential energy $U(a)$ (upper frame), in units of $(\hbar \omega) / 2$, as a function of the width $a$, and the corresponding phase plane defined by $\left(a_{t}, a\right)$. The parameters are dimensionless, with $P=-0.5495$ and $Q=0.0099$. $a$ is related to the meansquare radius as $\left\langle r^{2}\right\rangle=3 a^{2} / 2$.

As an example, in Table I we show one particular case where we have a phase transition, and also an analytical solution for one of the roots of Eq. (22), when $D=3$. We can observe that this condition is realized if $0>P \geqslant P_{c}$ $=-(1 / 8)^{(1 / 4)}$. When $Q=P^{8}$, the critical limit occurs for $P_{c}=-(1 / 8)^{(1 / 4)}$ and we have three roots for $a_{s}$, given by $a_{s 1}=a_{s 2}=\left|P_{c}\right|=0.5946$ and $a_{s 3}=0.4696$.

A typical plot of the potential $U(a)$ as a function of the width $a$, and the corresponding phase portrait defined in the plane $\left(a_{t}, a\right)$ for negative $\lambda_{2}$ and positive $\lambda_{3}$, is given in Fig. 1 for the case $D=3$ of a stable BEC. The parameters for the attractive cubic and repulsive quintic terms used in the figure are such that $n=1.948$ and $g_{3}=0.016$, corresponding to $P$ $=-0.5495$ and $Q=0.0099$. The number of atoms corresponds to the situation in which we have two minima with the same value for $U(a)$. From the variational expressions given in this section and our mechanical analogy, one should observe that, in the pictorial example of Fig. 1, if we have a solution located at the right minimum (for example) it cannot migrate to the left minimum, unless the chirp parameter corresponds to an energy greater than the difference between the right minimum and the maximum that is in between.

\section{ANALYSIS OF THE OSCILLATIONS OF THE BEC IN THREE DIMENSIONS}

The different dynamical regimes in the condensate oscillations can be described by the cross sections of the curve of $U(a)$ with the levels of the effective total energy. Following our mechanical analogy, we have

$$
H\left(P_{R}, R\right)=\frac{P_{R}^{2}}{2 m}+U_{P}(R),
$$

where $P_{R}$ is the momentum conjugate to $R$, given in Eq. (14). In our dimensionless variables, the corresponding initial Hamiltonian is given by

$$
H_{0}=\left.\frac{3}{2}\left(a_{t}\right)^{2}\right|_{0}+U\left(a_{0}\right)=\left(\frac{3}{2}\right) a_{0}^{2} b_{0}^{2}+U\left(a_{0}\right) \text {, }
$$

where Eq. (9) was used. Thus, by varying the initial conditions $a_{0}$ and $b_{0}$, we alter $H_{0}$.

This case was recently analyzed using the moments methods in Refs. $[1,8,15]$, under some specific assumptions. The assumption $\left.a_{t}\right|_{0}=0$ was considered in Refs. [1,15], and $\left.a_{t}\right|_{0} \neq 0$ in Ref. [8]. In Ref. [15], a generalization of the Weinstein criterion for the collapse [14] was obtained, in the case of three dimensions, and when there is only cubic term in the NLSE.

It will be interesting to derive criteria for stability more generally applied, using a variational approach and by comparison with results obtained with numerical simulations. For this purpose, we should first obtain expressions for the meansquare root that extremizes the total energy. In the variational approach, for $D$ dimensions, the equations for the total energy and chemical potential are given by Eqs. (20) and (21). So, where $D=3$, the equations corresponding to Eqs. (22), (23), and (24) are

$$
\begin{gathered}
a_{s}^{8}-a_{s}^{4}-P a_{s}^{3}-Q=0, \\
U\left(a_{s}\right)=2 a_{s}^{2}+\frac{1}{a_{s}^{2}}+\frac{P}{2 a_{s}^{3}}=\frac{5 a_{s}^{2}}{2}+\frac{1}{2 a_{s}^{2}}-\frac{Q}{2 a_{s}^{6}}, \\
\mu\left(a_{s}\right)=3 a_{s}^{2}+\frac{P}{2 a_{s}^{3}}=\frac{7 a_{s}^{2}}{2}-\frac{1}{2 a_{s}^{2}}-\frac{Q}{2 a_{s}^{6}} .
\end{gathered}
$$

Here we should note that an exact expression for the Hamiltonian, corresponding to the VA given by Eqs. (20), can be derived for the general case. This will be useful for the exact numerical calculations that we are going to perform in three dimensions. We consider the following scaling in the wave function $\psi$ of the system [24]:

$$
\psi=\alpha^{-3 / 2} \chi(\vec{\xi}) \quad \text { with } \quad \vec{\xi} \equiv \frac{\vec{r}}{\alpha}
$$

Then, for the total Hamiltonian, we obtain

$$
H(\alpha)=\frac{X_{s}}{\alpha^{2}}+\alpha^{2}\left\langle\xi^{2}\right\rangle+\frac{\lambda_{2}}{2} \frac{Y_{s}}{\alpha^{3}}+\frac{\lambda_{3}}{3} \frac{Z_{s}}{\alpha^{6}},
$$

where

$$
\begin{gathered}
X_{s} \equiv-\int \chi^{\dagger} \Delta \chi d^{3} \xi, \quad\left\langle\xi^{2}\right\rangle=\int \xi^{2}|\chi|^{2} d^{3} \xi \\
Y_{s} \equiv \int|\chi|^{4} d^{3} \xi, \quad Z_{s} \equiv \int|\chi|^{6} d^{3} \xi .
\end{gathered}
$$




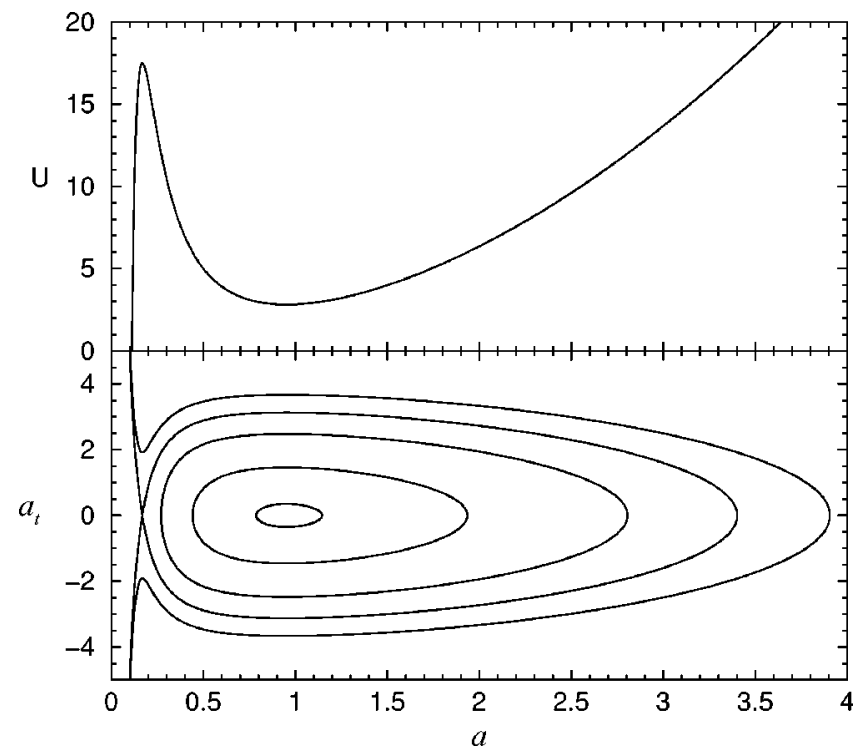

FIG. 2. For $\lambda_{2}<0$ (with $n=0.6$, or $P=-0.1692$ ) and $\lambda_{3}$ $=0(Q=0)$, as in Fig. 1, we show a variational plot of $U(a)$ (upper frame), as a function of the width $a$, and the corresponding phase plane defined by $\left(a_{t}, a\right)$.

By taking $\delta H(\alpha) /\left.\delta \alpha\right|_{\alpha=1}=0$, we obtain the characteristic equation

$$
X_{s}-\left\langle r^{2}\right\rangle+\frac{3}{4} \lambda_{2} Y_{s}+\lambda_{3} Z_{s}=0
$$

With the above equation for the energy, we have

$$
H=\frac{2 X_{s}}{3}+\frac{4}{3}\left\langle r^{2}\right\rangle-\frac{\lambda_{2}}{4} Y_{s} .
$$

Correspondingly, for the chemical potential, we obtain

$$
\mu=2\left\langle r^{2}\right\rangle+\frac{\lambda_{2}}{4} Y_{s} .
$$

It is interesting to observe that when the two-body interaction is zero, the exact result for the chemical potential is twice the mean-square radius, as shown above [in agreement with the variational result of Eq. (29), where $\left.a_{s}^{2}=2 / 3\left\langle r^{2}\right\rangle\right]$. As specific cases will be considered in the next subsections, we will discuss this point in more detail. For now, we can use the specific example of $Q=0$, represented in Fig. 2, in order to illustrate the validity of the general criterion (also valid for $Q \neq 0$ ) that we consider. In this case, we have just one minimum and a maximum of $U(a)$.

A given chirp $b(t)$ in the wave function corresponds to the square root of the kinetic energy, given by the Hamiltonian in Eq. (26). With a small initial chirp, we should have oscillations around the minimum of $U(a)$. The square of the chirp must be larger than the difference between the minimum and maximum of the effective potential energy $U(a)$ for the system to become unstable. So, from Eq. (26), and given that $H_{0}$ is conserved, we can derive a minimum criterion for stability. The given kinetic energy (related to the chirp) must be smaller than the corresponding variation of the potential energy, in order to maintain stable oscillations around the minimum (where $a=a_{s 1}$ ). The initial chirp $b_{0}$ must satisfy the condition

$$
\begin{gathered}
b_{0}^{2} a_{s 1}^{2} \leqslant-\frac{2}{3} \Delta U(a), \\
b_{0}^{2} \leqslant \frac{2}{3} \frac{U\left(a_{s 2}\right)-U\left(a_{s 1}\right)}{a_{s 1}^{2}},
\end{gathered}
$$

where $a_{s 2}$ is the value of $a$ corresponding to the maximum of $U(a)$, and $a_{s 1}$ corresponds to the minimum of $U(a)$. The maximum initial chirp is given by

$$
b_{0, m}=\sqrt{\frac{2}{3}} \frac{\sqrt{U\left(a_{s 2}\right)-U\left(a_{s 1}\right)}}{a_{s 1}} .
$$

From the above equation, we should also note that the critical values for the number of atoms, related to the parameters $P$ and $Q$, can be obtained by the condition that $U\left(a_{s 2}\right)$ $\rightarrow U\left(a_{s 1}\right)$. We should also observe that the chirp parameter is introduced in the exact wave function by an exponential factor, as in the VA [see Eq. (4)].

The frequency of the linear collective oscillations, $\omega_{L}$, is another relevant quantity with which to analyze the stability of the condensate. When the system becomes unstable, it reaches the value zero. From the radial variation of the force around a minimum $a_{s 1}$, obtained from Eqs. (27) and (28), $\omega_{L}$, in units of the trap frequency $\omega$, is given by

$$
\begin{aligned}
\omega_{L}=\sqrt{\left.\frac{1}{3} \frac{d^{2} U}{d a^{2}}\right|_{a=a_{s 1}}} & =\sqrt{5-\frac{1}{a_{s 1}^{4}}+\frac{3 Q}{a_{s 1}^{8}}} \\
& =\sqrt{8-\frac{4}{a_{s 1}^{4}}-\frac{3 P}{a_{s 1}^{5}}} .
\end{aligned}
$$

One of the requirements for the instability of the BEC is that $\omega_{L}$ is zero, when the minimum and maximum of $U$ disappear. So it is convenient to analyze the critical points when the system becomes unstable; at such points, the system collapses (if we have only one minimum) or one of the phases (characterized by a minimum) disappears. From Eq. (28),

$$
\begin{aligned}
\left.\frac{1}{3} \frac{d^{2} U}{d a^{2}}\right|_{a=a_{s}}= & \frac{5 a_{s}^{8}-a_{s}^{4}+3 Q}{a_{s}^{8}} \\
& =\frac{8 a_{s}^{5}-4 a_{s}-3 P}{a_{s}^{5}} .
\end{aligned}
$$

We can only have two minima (given by $a_{s 1}$ and $a_{s 3}$ ) and one maximum (at $a_{s 2}$ ) for negative $P$ and positive $Q$. In this case, two critical limits are possible, corresponding to the situations in which the position of one of the minima, $a_{s 1}$ or $a_{s 3}$, is equal to the position of the maximum (one of the phases disappears). Note that in the critical limit we have 
$a_{\min }=a_{\max }=a_{c}$. In the next two subsections, we will consider particular cases in which we have only one nonlinear term present in the NLSE: a cubic term or a quintic term. The discussion of more general cases, where both terms are present in the NLSE, we leave for Sec. IV.

\section{A. Case of $Q=0$, with attractive two-body term}

The roots for the maximum $\left(a_{s 2}\right)$ and the minimum $\left(a_{s 1}\right)$ of $U$ can be obtained from Eq. (27):

$$
a_{s}^{5}-a_{s}+|P|=0 .
$$

Combining this equation with Eq. (40), we have

$$
\left.\frac{1}{3} \frac{d^{2} U}{d a^{2}}\right|_{a=a_{s}}=5-\frac{1}{a_{s}^{4}} .
$$

In the critical limit $[31,5]$,

$$
\begin{gathered}
a_{c}=\left(\frac{1}{5}\right)^{1 / 4}, \quad P_{c}=-\frac{4}{5}\left(\frac{1}{5}\right)^{1 / 4}=-0.53499, \\
n_{c}=2 \sqrt{\pi}\left|P_{c}\right|=1.8965 .
\end{gathered}
$$

where Eqs. (41) and (13) were used. With the above root of Eq. (41), the other roots can also be easily found numerically for any value of $P$. Given $P$, once we obtain the positions of the minimum $\left(a_{s 1}\right)$ and maximum $\left(a_{s 2}\right)$ of $U$, we can calculate the value of the maximum initial chirp for the collapse, using Eq. (38):

$$
b_{0, m}=\sqrt{\frac{a_{s 1}^{2}-a_{s 2}^{2}}{3 a_{s 1}^{4} a_{s 2}^{2}}\left(1-5 a_{s 1}^{2} a_{s 2}^{2}\right)} .
$$

Our results, when we have only the cubic term in the nonlinear effective potential $(Q=0)$, are shown in Figs. 2 and 3. In Fig. 2, for $P=-0.1693$ (corresponding to $n=0.6$ ), in the lower frame we show the phase space given by $a_{t}$ versus $a$. In the upper frame, we have the total energy, in units of $N(3 / 2) \hbar \omega$, as a function of $a$.

In Fig. 3, in the lower frame we show our results for the maximum initial chirp $b_{0, m}$, as a function of $n$, for stable solutions. $n$ is the reduced number of atoms, given by Eq. (13). In this figure, we also represent the total energy (upper frame) and the average of the square radius, $\left\langle r^{2}\right\rangle$, as a function of $n$.

The static variational expression for the maximum initial chirp was derived from Eq. (9) and energy conservation requirements given by Eq. (37). The circles correspond to results obtained by the time-dependent VA, by increasing the initial conditions for the chirp until the system collapses. We also obtain results for the maximum initial chirp using the same Eq. (37), considering exact PDE results for the observables presented in the equation. The results for the static case are represented by a solid line, and those for the timedependent case by squares. We observed small differences in the absolute values of $b_{0, m}$ when considering the trail $b_{0}$ as positive or negative. This fact can be qualitatively under-

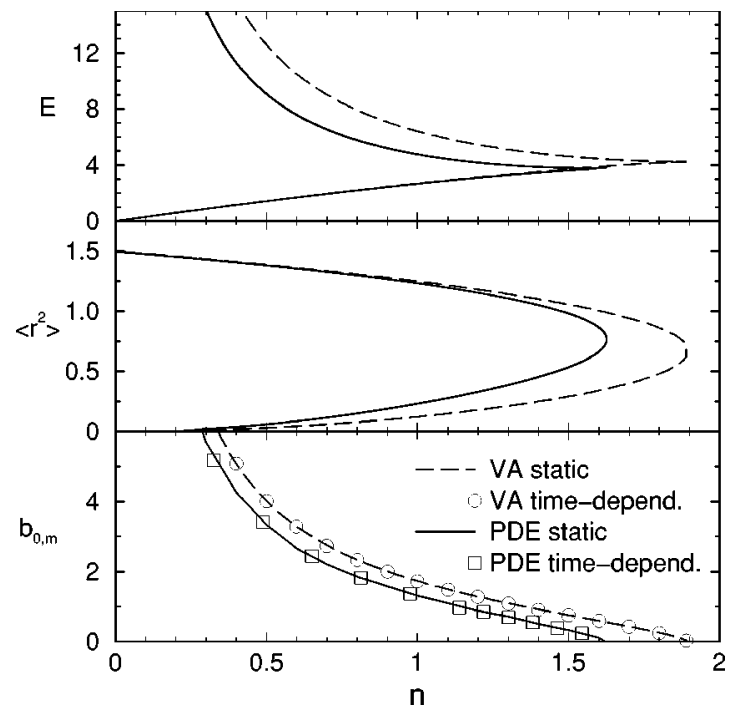

FIG. 3. Results for $Q=0$, as functions of $n$, the reduced number of atoms defined in the text. In the upper frame we have $E=n U$, the total energy in units of $(N / n)(\hbar \omega / 2)$; in the middle frame, the mean-square radius $\left\langle r^{2}\right\rangle$, in units of $\hbar /(m \omega)$; and, in the lower frame, the maximum initial chirp. In the critical limit, $n_{c}=1.8965$ and $P_{c}=-0.53499$, the total energy is given by $E=n U=n_{c} \sqrt{5}$ $=4.2407$, corresponding to $E_{T}=\sqrt{5} N_{c}(\hbar \omega / 2)$.

stood by a small dephasing of the wave that occurs near the wall of the oscillator, when it is bouncing back.

For the mean-square radius, we observe that the VA results approaches the exact PDE results in the unstable branch in the limit $n \rightarrow 0$. These results are consistent with results obtained in Ref. [24], where it was shown that, for a trapped NLSE with a cubic term, the unstable branch of the chemical potential diverges in the limit $n \rightarrow 0$, with $\left\langle r^{2}\right\rangle$ collapsing to zero (this limit is the nontrapped solution of the NLSE).

\section{B. Case of $\boldsymbol{P}=0$ with attractive three-body term}

As in Sec. III A, for a stable condensate the maximum initial chirp is given by Eq. (38), where the roots are given by Eq. (27). The real and positive roots correspond to stable solutions, such that only one is meaningful when $Q>0$. When $Q<0$, we obtain two real and positive roots of $a$, where $0>Q \geqslant-1 / 4$. These roots are

$$
a_{s 1 / s 2} \equiv a_{+/-}=\left[\frac{1}{2} \pm \sqrt{\frac{1}{4}+Q}\right]^{1 / 4},
$$

where the minimum of $U$ is given by $a_{+}\left(\equiv a_{s 1}\right)$, and the maximum by $a_{-}\left(\equiv a_{s 2}\right)$. The critical limit occurs at $a_{c}^{4}$ $=1 / 2$ or $Q_{c}=-\left|Q_{c}\right|=-1 / 4$. From Eqs. (45) and (40),

$$
\left.\frac{1}{3} \frac{d^{2} U}{d a^{2}}\right|_{a=a_{s}}= \pm \frac{2 \sqrt{1-4|Q|}}{|Q|}(1 \mp \sqrt{1-4|Q|}),
$$

and the frequency of the linear collective oscillations, $\omega_{L}$, near the minimum is given by 


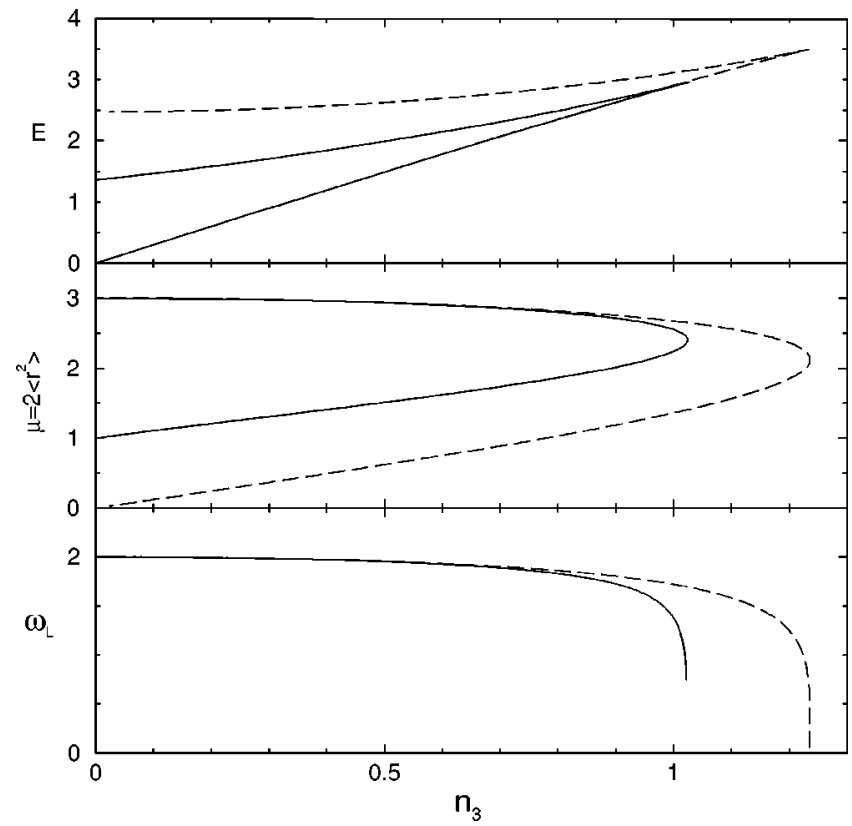

FIG. 4. Results for $P=0$, as functions of the reduced number of atoms $n_{3}$. The total energy is given in the upper frame, in units of $\left(N / n_{3}\right)(\hbar \omega / 2)$; in the middle frame we have the chemical potential in units of $(\hbar \omega) / 2$; and, in the lower frame, the frequencies of the collective breathing mode. In the VA, the critical limit for stability, $Q=Q_{c}=-1 / 4$, corresponds to $n_{3, c}=1.2371$, where $E=n_{3} U_{c}$ $=2 \sqrt{2} n_{3, c}=3.499$ [corresponding to $\left.E_{T}=2 \sqrt{2} N_{c}(\hbar \omega / 2)\right]$.

$$
\omega_{L}=\sqrt{\frac{2 \sqrt{1-4|Q|}}{|Q|}(1-\sqrt{1-4|Q|})} .
$$

In Fig. 4, we present the main results of this subsection with $P=0$. As noted in Eqs. (29) and (36) (the variational and exact expressions, respectively), the chemical potential is exactly twice the mean-square radius. In the plots, the VA results are shown by dashed lines and the exact PDE by solid lines. The variational critical limit $Q=Q_{c}=-1 / 4\left(a_{c}^{4}=1 / 2\right)$ corresponds to $n_{3, c}=1.2371$ where $n_{3}$ is the reduced number of atoms, that is given by the normalization of the wave function. In this case, Eq. (12) will give us the relations between $|Q|,\left|\lambda_{3}\right|$, and $n_{3}$ :

$$
n_{3}=\frac{\sqrt{\left|\lambda_{3}\right|} N}{2 \pi} \text { and }|Q|=\frac{8 n_{3}^{2}}{9 \pi \sqrt{3}} .
$$

Given $\lambda_{3}$, we can obtain the corresponding critical number of atoms,

$$
N_{c}=\frac{3 \pi^{3 / 2} 3^{1 / 4}}{2 \sqrt{2} \sqrt{\left|\lambda_{3}\right|}}=\frac{7.7729}{\sqrt{\left|\lambda_{3}\right|}},
$$

a result consistent with no limit in the number of atoms if $\lambda_{3} \rightarrow 0$.

In Fig. 4, we also observe that $\partial \mu / \partial n_{3}<0$ (branch of minima) and $\partial \mu / \partial n_{3}>0$ (branch of maxima) correspond, respectively, to stable and unstable solutions. We note that a localized stable structure can only exist for positive $\mu$. The

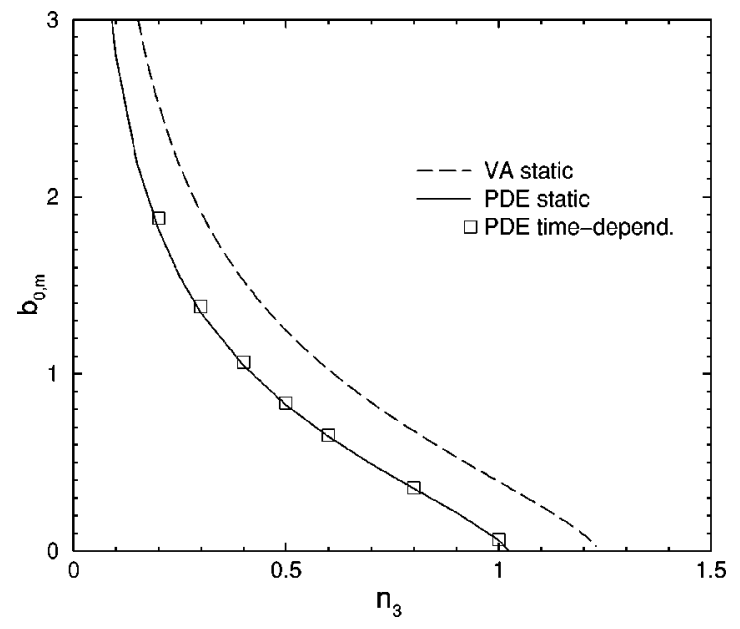

FIG. 5. Results for the maximum initial chirp $b_{0, m}$, considering $\lambda_{3}<0$ and $\lambda_{2}=0$, using the VA and exact PDE results.

results in this case, that the effective interaction contains only a trap and a quintic term, are in agreement with the VK criterion [22]. The observed exact relation between the chemical potential and the mean-square radius $\left(\mu=2\left\langle r^{2}\right\rangle\right)$, in the present case, $\lambda_{2}=0$, was proved in Eq. (36).

In the branch with unstable solutions, we observe numerically that in the limit $n_{3} \rightarrow 0$ the wave function approaches the form $\psi \propto \exp \left(-r^{2} / 2\right) / r$, with $\mu \rightarrow 1$ and $\left\langle r^{2}\right\rangle \rightarrow 1 / 2$. This solution is proved analytically to be an irregular solution of the oscillator [Recall that, when $\lambda_{2}=0$ and $\lambda_{3}=0$, in Eq. (2), the regular oscillator solution gives $\mu=3$; for the irregular solution in the origin we have $\mu=1$ in our energy unit $(\hbar \omega / 2)]$. Thus for unstable solutions, with small $|Q|$, the Gaussian ansatz (started by considering the exact regular solution of the oscillator in the limit $n_{3}=0$ ) fails. By keeping (artificially) the Gaussian shape for the unstable solutions, the radius is forced to be zero. This explains the discrepancy between the present VA and PDE results in the unstable region. A more appropriate ansatz for the trapped NLSE (when $\lambda_{2}$ and/or $\lambda_{3}$ is nonzero) could be built by considering the two (regular and irregular) solutions of the harmonic oscillator.

Even considering the discrepancy between VA and PDE results for unstable (maxima) solutions, there is a reasonable agreement for the results obtained for the maximum initial chirp $b_{0, m}$, as shown in Fig. 5. In Fig. 6, we have a variational result for the potential energy as a function of $a$, and the corresponding phase space, as in Figs. 1 and 2. Static predictions on the PDE are also in good agreement for the maximum initial chirp. In PDE calculations we have numerically verified small differences between negative and positive $b_{0, m}$, as in the case analyzed and explained in Sec. III A (related to Fig. 3). Using Eq. (38) with $P=0$, the maximum initial chirp is given by

$$
\begin{aligned}
b_{0, m} & =\sqrt{\frac{1}{3|Q|}(1-2 \sqrt{|Q|})[\sqrt{1-4|Q|}-1+2 \sqrt{|Q|}]} \\
& \approx \sqrt{\frac{2}{3 \sqrt{|Q|}}\left(1-\frac{3 \sqrt{|Q|}}{2}\right) \text { for } \quad|Q| \ll 1 .}
\end{aligned}
$$




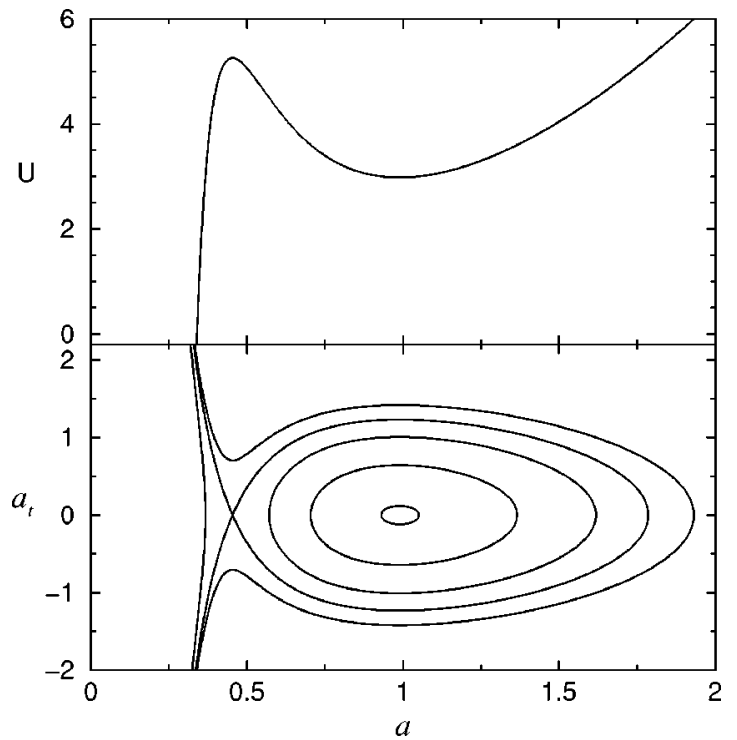

FIG. 6. For $\lambda_{3}<0$ and $\lambda_{2}=0 \quad(Q<0$ and $P=0)$, with $n_{3}$ $=0.5$, as in Figs. 1 and 2, we present results of the VA for the total potential energy $U$ as a function of $a$, and a plot of the phase space of $a_{t}$ vs $a$.

The above result shows that, when the three-body parameter is negative and small, the maximum initial chirp is $\propto|Q|^{-1 / 4}$, or $\propto\left(1 / \sqrt{n}_{3}\right)$, as shown in Fig. 5 .

\section{DYNAMICS OF BEC WITH TWO- AND THREE-BODY INTERACTIONS}

In this section, we analyze the cases where we have nonzero cubic $\left(\lambda_{2}\right)$ and quintic $\left(\lambda_{3}\right)$ interaction terms in the NLSE. The different possibilities relative to the signs of the two and three-body interactions are studied in the next three subsections, where we exclude one of the cases in which the signs are the same. When $Q$ has the same sign as $P$, the basic physical picture is not essentially altered in comparison to the cases already discussed, where one of these quantities is zero. However, it is worthwhile to examine the changes in the collective excitations when $\lambda_{2}$ and $\lambda_{3}$ are positive, as such observables can be useful to obtain information about possible manifestations of three-body interactions.

\section{A. Case of repulsive cubic and quintic interactions $\left(\lambda_{2}>0\right.$ and $\left.\lambda_{3}>0\right)$}

From Eq. (39), one should observe that the frequency of the collective oscillations will decrease as we increase $P$, or if the position of the minimum, $a_{s 1}$, decreases. As all quantities present in Eq. (39) are positive, this frequency in the VA has an upper limit at the value $2 \sqrt{2}$, such that a reasonable frequency of oscillations around the minimum will be smaller than that (also see Ref. [32]).

The effects of the unharmonicity of the oscillations can be taken into account by the expansion of the effective potential near the bottom $a_{c}$ on the power of deviations $y=a-a_{s 1}$. The equation for $y(t)$ is

$$
y_{t t}=-B_{1} y-B_{2} y^{2}-B_{3} y^{3},
$$

where

$$
\begin{aligned}
& B_{1}=1+\frac{3}{a_{s 1}^{4}}+\frac{4 P}{a_{s 1}^{5}}+\frac{7 Q}{a_{s 1}^{8}}, \\
& B_{2}=-\frac{6}{a_{s 1}^{5}}-\frac{10 P}{a_{s 1}^{6}}-\frac{28 Q}{a_{s 1}^{9}}, \\
& B_{3}=\frac{10}{a_{s 1}^{6}}+\frac{20 P}{a_{s 1}^{7}}+\frac{84 Q}{a_{s 1}^{10}} .
\end{aligned}
$$

Then, applying the perturbation techniques to the theory of nonlinear oscillations [25], we find the frequency of weakly nonlinear collective excitations,

$$
\omega_{N L}=\sqrt{B_{1}}\left[1+\left(\frac{3 B_{3}}{8 B_{1}}-\frac{5 B_{2}^{2}}{12 B_{1}^{2}}\right) \sigma^{2}\right],
$$

where $\sigma$ is the amplitude of the oscillations. The correction to the linear frequency is proportional to the square of the amplitude of oscillations of the condensate.

The above estimate of the nonlinear oscillations in BEC, when both the two- and three-body terms are positive, can be a relevant piece of information to determine a possible manifestation of three-body interaction in the condensates, and its magnitude. We should observe that recently, in Ref. [26], an observation was reported of nonlinear oscillations in BEC's of a gas with rubidium atoms.

Let us estimate the amplitude of oscillations of the width of the atomic cloud. The points of maximum and minimum width are defined by the section points of the line $H_{0}$ $=$ const with the potential curve $U(a)$. For large width, $a$ $\gg 1$, we can obtain the estimate

$$
\sigma_{ \pm}=\sqrt{\frac{H_{0}}{3} \pm \sqrt{\left(\frac{H_{0}}{3}\right)^{2}-1}}
$$

where $\sigma_{+(-)} \equiv \sigma_{\max (\min )}$. It is natural to assume that the asymptotic value of the width is the averaged value between $\sigma_{\max }$ and $\sigma_{\min }$.

\section{B. Case of attractive cubic and repulsive quintic interactions $\left(\lambda_{2}<0\right.$ and $\left.\lambda_{3}>0\right)$}

In this case, there is no collapse, but the condensate can have up to two distinct phases when $\left|\lambda_{2}\right|$ is smaller than a critical value. From the beginning, when $\lambda_{3}=0, U$ has one minimum at $a=a_{s 1}$ and a maximum at $a=a_{s 2}$. By considering a fixed positive (and small) $\lambda_{3}$, a second minimum of $U\left(a_{s 3}\right)$ appears, corresponding to a denser phase in the condensate. As we increase the value of $|P|$, we can reach a critical value where the minimum of the normal phase disappears [3].

When $H_{0} / 3<\Delta U=\left|U\left(a_{s 2}\right)-U\left(a_{s}\right)\right|$, with $a_{s}=a_{s 1}$ or $a_{s 3}$, we have small-amplitude oscillations near the fixed point $a_{s}$. With $H_{0} / 3>\Delta U$, the character of the oscillations changes, and we have large-amplitude oscillations, due to the 
motion of the effective particle between the wall given by the repulsive three-body interaction and the other curve given by the quadratic potential. With $a_{s}$ close to $a_{s 3}$ and $H_{0} / 3<\Delta U_{2}=\left|U\left(a_{s 2}\right)-U\left(a_{s 3}\right)\right|$, the condensate oscillates with an amplitude restricted from below by $a_{s 2}$.

The position of the minimum $a_{s 1}$ of $U(a)$ is defined by the equilibrium between two- and three-body interactions; the positions of the other extrema of $U$, the fixed points $a_{s 2}$ and $a_{s 3}$, are mainly defined by the contributions of the twoand three-body terms. These considerations allow us to obtain analytical expressions for such fixed points, that are given by the real and positive roots of the equation given by the first derivative of $U(a)$.

In order to analyze the frequencies of the linear oscillations [given by Eq. (39)], we return to Eq (40), where $P$ $=-|P|$ and $Q=|Q|$. The physically relevant situations occur when $|P|$ is a fraction of 1 and $Q \ll 1$. Of particular interest are the cases where two phases (corresponding to two minima) are possible, at $a=a_{s 1}$ and $a=a_{s 3}$.

Let us consider, for example, the particular case examined in Table I, that we have a one-point solution given by $a_{s 1}$ $=|P|$ and $Q=a_{s 1}^{8}=|P|^{8}$. This example is chosen not only for convenience but because it is not far from a more realistic situation. Usually we scale the normalization of the wave function with a value of $n$ that is directly proportional to $|P|$. Thus the three-body parameter $\lambda_{3}$ is related to the particular point we are considering. The critical limit in this case is given by $\left|P_{c 1}\right|^{4}=(1 / 8)\left(\left|P_{c 1}\right|=0.5946\right)$ and $Q_{c 1}=1 / 64$, as shown in Table 1 , corresponding to $n_{c 1}=2.1078$ and $g_{3}$ $=0.021$ [see Eq. (13)],respectively. At such a critical limit the frequency of the linear oscillations around the minimum $a_{s 1}$ goes to zero [In a practical situation, before the system reaches this critical limit in the normal phase, it tunnels to a denser phase, when both energies are equal]. With $|P|$ larger than $\left|P_{c}\right|$, there is only one phase (minimum) corresponding to a denser phase. As we reduce the value of $|P|$, we reach a second critical point at $a_{s 2}=a_{s 3}=\left|P_{c 3}\right|$, where $a_{s 3}$ is the position of the minimum of the denser phase. A given value of $|P|$ should be between the limits $\left|P_{c 3}\right|<|P|<\left|P_{c 1}\right|$ for the existence of two phases.

Thus, for the normal phase, we note that the corresponding frequencies of the oscillations are approximately given by the harmonic oscillator, with $\omega_{L 1} \sim 2$ (twice the trap frequency). This can be seen in previous cases, where one of the nonlinear terms is zero and the other is small.

Consider the case exemplified in Fig. 1, where $P$ $\approx-0.55(n \approx 1.95)$ and $Q \approx 0.01 \quad\left(g_{3}=0.016\right)$, and the corresponding roots for the minima are $a_{s 3} \approx 0.386$ and $a_{s 1}$ $\approx 0.726$. Using Eq. (39), we can observe that the frequency of the oscillations in the denser phase, $\omega_{L 2}=4.493$, is much larger than the frequency of the normal phase, $\omega_{L 1}=1.339$. If we take more realistic parameters like for example, $g_{3}$ $=0.001$ and $n=1.5$, using Eqs. (13) and (27), we obtain $a_{s 1}=0.840$ (minimum of the "gas" phase), $a_{s 2}=0.434$ (maximum), and $a_{s 3}=0.105$ (minimum of the "liquid" phase). The corresponding frequencies of the linear oscillations of the two phases are $\omega_{L 1}=1.73$ and $\omega_{L 2}=258$. So, in general, we observe that it should be expected that

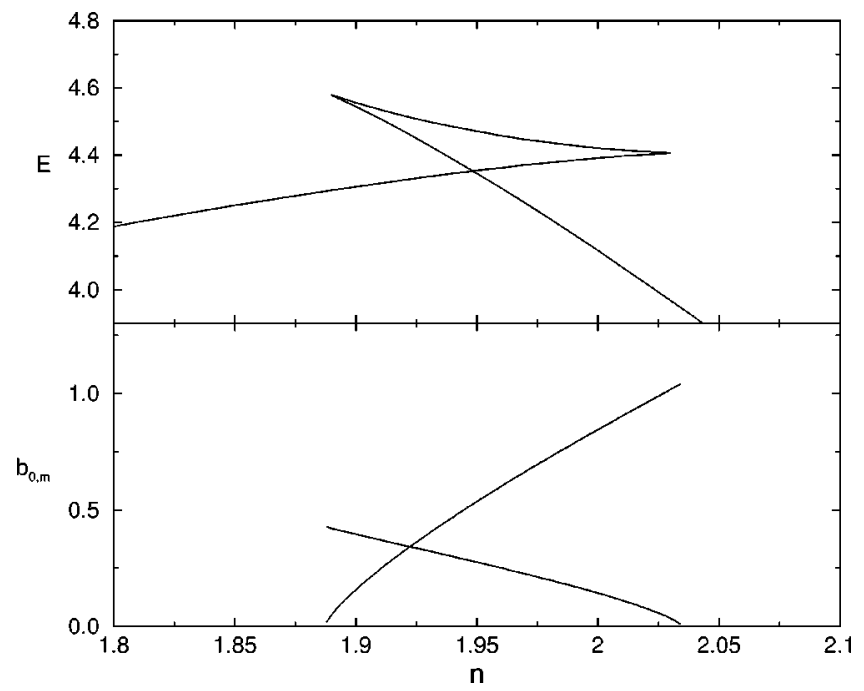

FIG. 7. VA results for the total energy and for the corresponding maximum initial chirp, as functions of the reduced number of atoms $n$, when we have an attractive two-body interactions and a fixed repulsive three-body interaction with $g_{3}=0.016$.

$$
\omega_{L 2} \gg \omega_{L 1}
$$

This unequality for the frequencies was also confirmed in numerical PDE calculations done in Ref. [3].

The amplitude of the nonlinear oscillations in the dense phase can be found from the observation that $a_{s 3} \ll 1$. Then terms with $a^{-6}$ and $a^{-3}$ are dominant in the effective potential energy, and an approximate solution can be found:

$$
\sigma_{\max , \min }=\left(\frac{|P|}{2\left|H_{0}\right|} \pm \sqrt{\frac{P^{2}}{4 H_{0}^{2}}-\frac{Q}{2\left|H_{0}\right|}}\right)^{1 / 3} .
$$

An interesting phenomenon occurs when the initial $\left.a_{t}\right|_{0}$ $=a_{0} b_{0}$ is large. Then the character of the cloud oscillations will change, from oscillations near $a_{s 1}$ and $a_{s 3}$ to largeamplitude oscillations defined mainly by the quadratic potential (see Fig. 1). Let us estimate the criterion for the bifurcation phenomenon in the oscillations. Taking into account the expression for the energy $H_{0}$, the bifurcation point is given by

$$
b_{0, m} \geqslant \sqrt{\frac{2 \Delta U}{3}} \frac{1}{a_{s 1}} .
$$

The result is shown in Fig. 7, using our Gaussian VA, which gives us a qualitative picture of the exact results that were already presented in Ref. [3].

In Fig. 8 we show a full numerical PDE calculation for a system beginning in the gas phase, which corresponds to the right minimum of the Fig. 1. Our results show that for a small chirp $\left(b_{0} \leqslant 0.10\right)$, the mean-square radius oscillates with small amplitude, according to its collective frequency. Applying a stronger chirp, near $b_{0} \geqslant 0.11$, we observe transitions back and forth between two phases, characterized by different amplitudes of the oscillations. As $b_{0}$ is large enough we observe that the oscillation pattern remain almost 


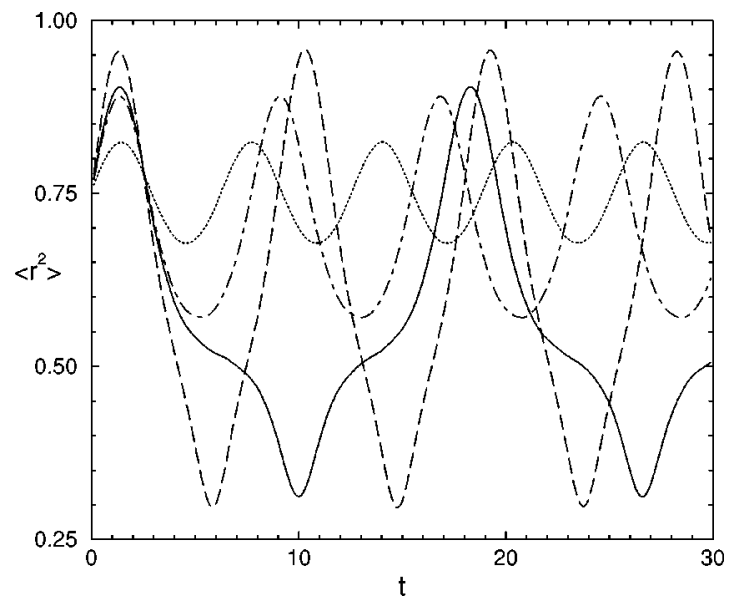

FIG. 8. Time evolution of the square radius with the full PDE in the case of a phase transition for $n=1.754$ and $g_{3}=0.016\left(\lambda_{2}\right.$ $<0, \lambda_{3}>0$ ), beginning in the gas (less dense) phase solution. The initial conditions (chirp) considered are $b_{0}=0.05$ (dotted line), $b_{0}$ $=0.10$ (dot-dashed line), $b_{0}=0.11$ (solid line), and $b_{0}=0.15$ (long dashed line).

fixed for large amplitude. A similar picture can be seen if we begin in the denser phase, with different initial conditions given by the chirp. These results were obtained by using the exact PDE calculation, and they are in qualitative agreement with the VA approach. The quantitative agreement between the VA and the exact PDE deviates considerably in the phase transition region. This should be expected, as we are far from the harmonic-oscillator behavior, in a region where an improvement in the ansatz is necessary.

\section{Case of repulsive cubic and attractive quintic interactions $\left(\lambda_{2}>0\right.$ and $\left.\lambda_{3}<0\right)$}

As both two- and three-body nonlinear terms are nonzero and have opposite signs (with attractive three-body and repulsive two-body interactions), one could expect a behavior similar to the case that was analyzed in Sec. IV B, and also represented in Fig. 1 (where the two-body interaction is attractive and the three-body interaction is repulsive). But contrary to such expectation, this case shows a different behavior for a small radius, and no phase transition is possible. We observe that in this case the system can collapse, as the behavior for a small radius is dominated by the three-body term, which is negative. This is represented in Fig. 9, in a variational plot of $U(a)$ versus $a$, together with a corresponding plot for the phase space.

In Fig. 10, we present the breathing-mode collective excitations for a few values of $g_{3}$ calculated in the variational approximation. The collective excitations show that even for small negative $g_{3}$ a limited number of atoms is allowed in the condensate. Now only a region of stationary condensate - the denser phase-exists. Two points exist that extremize the energy: one, $a_{s 1}$, is stable; and the other, $a_{s 2}$, is unstable. Here the situation is similar to the cases where we have a single attractive nonlinear term (cubic or quintic); the condition for collapse, in terms of the initial chirp $b_{0}$, is such that it must be larger than $b_{0, m}$ given in Eq. (38).

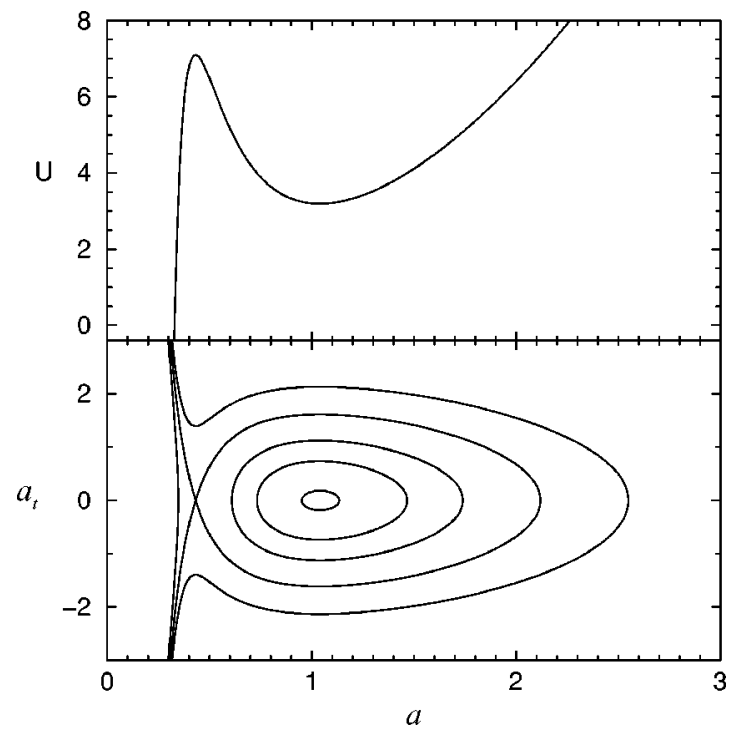

FIG. 9. As in Figs. 1, 2 and 5, in the upper frame we have the potential energy $U(a)$, and, in the lower frame, a plot of the corresponding phase space of $a_{t}$ vs $a$. We have nonzero values for both two- and three-body parameters, with $\lambda_{2}>0$ and $\lambda_{3}<0 \quad\left(g_{3}\right.$ $=-0.5)$.

One should note that this particular case $\left(\lambda_{2}>0\right.$ and $\lambda_{3}$ $<0)$ can be relevant in an analysis of experiments with BEC's performed with atomic systems that have repulsive two-body interactions. No collapse is expected if a real threebody effect is not manifested, or if the possible three-body effect is also repulsive. However, an attractive three-body effect will change this scenario, as the system must collapse for a certain critical maximum number of atoms. In this perspective, the present analysis shows that experiments with BEC's may be useful in detecting negative three-body forces. The corresponding maximum critical number of atoms for the stability of the condensate can also be obtained

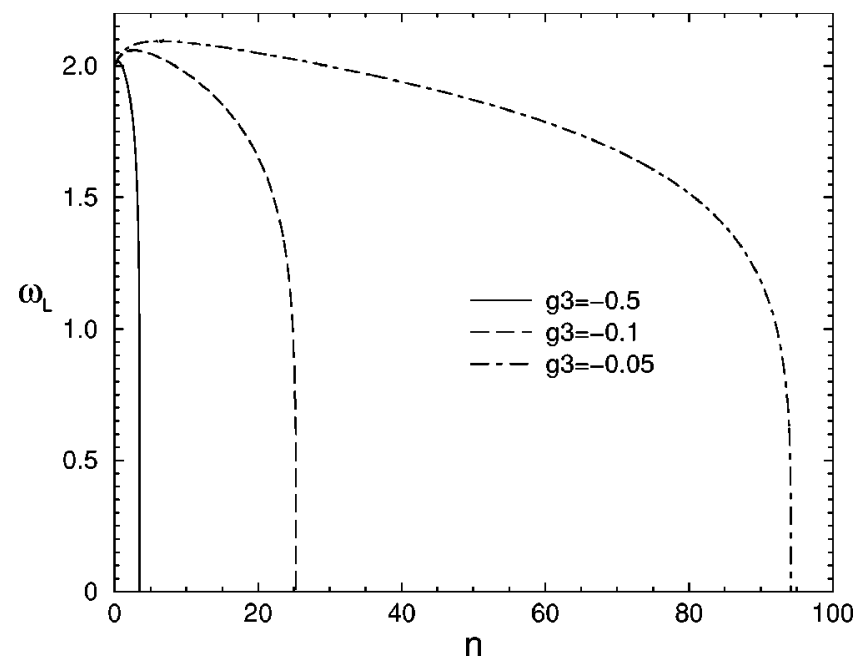

FIG. 10. Collective excitations for the breathing mode of the condensate for a positive two-body interaction $\left(\lambda_{2}>0\right)$, and for a set of negative three-body interactions $\left(\lambda_{3}<0\right)$, as shown inside the figure. 

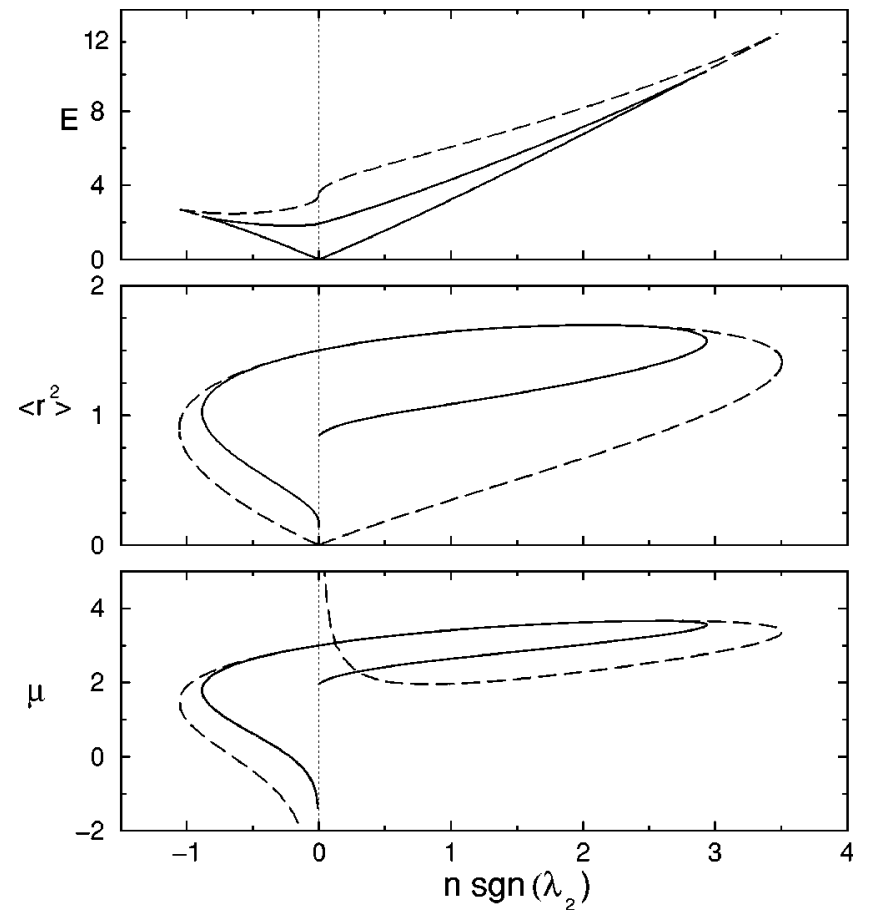

FIG. 11. Total energy $E$, mean-square radius $\left\langle r^{2}\right\rangle$, and chemical potential $\mu$, for negative $\lambda_{3}$ (with $g_{3}=-0.5$ ). The dashed line corresponds to the variational approach, and the solid line to exact numerical calculations. Departing from $\mu=3$, to the right or left, we have solutions corresponding to the minima of the total energy, until critical values $n_{c}$ (in both sides) are reached. After the critical numbers, the curves follow lower branches, corresponding to maxima.

from the present approach, by taking the limit $b_{0, m} \rightarrow 0$ (or $\left.a_{s 2} \rightarrow a_{s 1}\right)$. For instance, when $g_{3}=-0.5$, the critical number $n$ can be obtained from the right-hand side (positive $\lambda_{2}$ ) of Fig. 11.

In Fig. 11 we present, for a fixed attractive three-body interaction with $g_{3}=-0.5$, the total energy $E$, the meansquare-radius $\left\langle r^{2}\right\rangle$, and the chemical potential, as functions of the reduced number of atoms $n$ multiplied by the sign of the two-body interaction. We consider both negative $\left(\lambda_{2}\right.$ $<0)$ and positive $\left(\lambda_{2}>0\right)$ two-body interactions, and the value of $g_{3}=-0.5$ was chosen for numerical convenience. The variational approach (dashed line) gives a good description of the minima for small $|n|$, but fails for the maxima solutions, as one can see in the figure when comparing with the exact results (solid lines). In agreement with the variational results of collective excitations, the number of atoms allowed is limited to a critical number $n_{c} \sim 3.5$ (the corresponding exact result is $\sim 2.93$ ). For a more realistic value of the three-body parameter, with $g_{3}=-0.01$, the exact critical number is $n_{c} \sim 4200$.

In the upper frame of Fig. 11 we show a similar pattern for the minimum and the maximum of the energy, as already described with $\lambda_{3} \geqslant 0$ when $\lambda_{2}<0$ and with $\lambda_{3}>0$ when $\lambda_{2}=0$. Such results also indicate that an initial wave function with a larger enough chirp can make the condensate unstable, and that the corresponding magnitude of $b_{0, m}$ can be inferred from the absolute difference between the maxi- mum and minimum of the energy. This implies that, when we have a positive two-body interaction $\left(\lambda_{2}>0\right)$, the presence of a negative three-body term $\left(\lambda_{3}<0\right)$ can be detected in principle even if a number of atoms has not achieved a critical number. The initial maximum chirp plays a relevant role in this case.

\section{VK CRITERION}

In the context of stability analysis of NLSE bound states, the orbital stability, or stability with respect to the form, was first settled by the so-called Vakhitov-Kolokolov criterion [22], that was detailed in a recent review in Ref. [12]. A solitary wave is orbitally stable if the initial orbit, chosen near the ground-state orbit, implies that the orbit of the solution at any $t>0$ remains close to the ground state. The criterion was first demonstrated in Ref. [33], by minimizing the deviations between the orbital states and the ground state with respect to the initial position parameters. In our notation, the VK criterion for stability can be written as ${ }^{1}$

$$
\frac{\partial N}{\partial \mu}<0
$$

However, the original VK criterion and the above-cited demonstration only considered cases without trap, with nonlinearities expressed by $f\left(|\psi|^{2}\right)$. More recently, a general condition that guarantees the orbital stability of stationary trapped condensates, described by the NLSE with a cubic term, was formally demonstrated by Bergé et al. [24], in agreement with results analyzed in Ref. [7] for the case when the cubic term is negative.

The validity of the VK criterion appears to be solidly consolidated by the above-described formal demonstrations, and by the specific cases that have been considered. These results appear to support an extended range of applicability of the VK criterion to the NLSE. In the several cases that we considered in the present paper (by an exact numerical procedure and also by a Gaussian variational approach), the applicability of the VK criterion is out of the question in the cases that have been examined by other authors. We have also confirmed the applicability of the criterion in the case when we have only a trap and a quintic term in the effective interaction, as shown in Fig. 4.

The validity of the VK criterion cannot be extended to a trapped NLSE with two- and three-body terms, when the two-body term is negative, as one can observe from the results obtained in Ref. [3]. This is an unexpected result, considering that the criterion is applicable for nontrapped systems (with cubic and/or quintic terms), and also for the trapped cases when we have only the cubic or the quintic terms (and also in the cases that both terms have the same sign). The VK criterion is also not applicable when $\lambda_{2}>0$, as observed in Fig. 11, on the right side of the plot. The criterion fails particularly in the region near the oscillator solution (where $\mu=3$ ), where we can observe that $\partial \mu / \partial n$

\footnotetext{
${ }^{1}$ We should note that $\mu$ corresponds to $-\lambda$ of Ref. [12].
} 
$>0$ in a stable branch (upper) and also in the unstable branch (lower). (We recognize the stable branch as the one that corresponds to minima of the energy.) Therefore, in the present work, we confirm the conclusion about the limitation of the VK criterion: The nonvalidity of the VK criterion is verified numerically when we have a positive harmonic trap, with two- and three-body nonlinear terms with opposite signs.

\section{CONCLUSIONS}

The stability of a trapped condensate, with two- and threebody nonlinear terms, was studied in the present work, considering several aspects, as the initial conditions in the wave function and the validity of the Vakhitov-Kolokolov criterion. For the initial conditions we considered a chirp parameter, which is related to the initial focusing (defocusing) of the cloud. A nonzero initial chirp $b_{0}$ introduces oscillations in the condensate near the minimum of the energy, such that it can lead a previously stable system to collapse, when $b_{0}$ reaches a maximum limit $b_{0, m}$ that corresponds to the energy difference between a minimum and a maximum of the total potential energy. So, in the presence of a fixed value of the chirp, the number of atoms of a stable condensate with an attractive interaction is smaller than the corresponding value when the chirp is zero. When the potential has two minima (when cubic and quintic terms are present in the NLSE), related to two phases of the condensate, a bifurcation phenomenon is predicted depending on the value of the initial chirp. A maximum initial chirp can affect the system in such a way that the oscillations can switch from a gas phase to a liquid phase, and vice versa.

The present study was performed by using exact numerical solutions of the partial differential equation, as well as by a corresponding variational approach. Analytical predictions based on the time-dependent VA, using a Gaussian ansatz, were qualitatively confirmed by the exact time-dependent numerical simulations. We would like to point out the advantages of the Gaussian VA when comparing with the moments method, which encounters some difficulties in deriving collapse conditions even in the pure attractive two-body case, for some classes of the initial data (the sign of the timedependent function in the equation for the mean-square radius, $\left\langle r^{2}\right\rangle$, is not defined [8]).
The VA starts to deviate from the exact results when the system is close to collapse conditions, and particularly for unstable solutions (maxima) of the total energy. For these regions, where the Gaussian VA fails, one should improve the ansatz or take the results as a qualitative picture to guide the exact numerical calculations. In this respect, one should note that, for a NLSE without a trap potential, it is well known that the time-dependent variational approach fails to describe the region near the collapse $[12,13]$.

Considering that in this work we have shown the relevance of an initial chirp parameter to study the stability of the condensate, we should observe that a good estimate for such a parameter relies on a good approach to determing both minimum (stable) and maximum (unstable) solutions for the total energy. The Gaussian VA is in good agreement (quantitatively) with exact results for the minima, but can only give a qualitative description for the maxima. Still we can observe that the VA calculations of the chirp parameter, when compared with exact numerical calculations, are in reasonable agreement. We also would like to point out that the present approach can be extended to study the stability of chirped laser beams in inhomogeneous three dimensional media with Kerr nonlinearity.

Finally, the main results of the present work are as follows: (i) A chirp parameter in the wave function was shown to be useful to study the initial conditions for a stable condensate to remain stable. (ii) We showed that the VK criterion cannot be extended to cases of a harmonic trapped $\mathrm{BEC}$ when the nonlinear two- and three-body terms have opposite signs. (iii) If an atomic system has a repulsive two-body interaction, collapse is possible if the effective three-body interaction is negative. By this perspective, one can use the observed critical number of atoms in order to determine the corresponding three-body parameter.

\section{ACKNOWLEDGMENTS}

We are grateful to Fundação de Amparo à Pesquisa do Estado de São Paulo (FAPESP-Brazil) for partial financial support. L.T. and T.F. also acknowledge partial support from Conselho Nacional de Desenvolvimento Científico e Tecnológico.
[1] L.P. Pitaevskii, Phys. Lett. A 221, 14 (1996); F. Dalfovo, S. Giorgini, L.P. Pitaevskii, and S. Stringari, Rev. Mod. Phys. 71, 463 (1999).

[2] C.A. Sackett, H.T.C. Stoof, and R.G. Hulet, Phys. Rev. Lett. 80, 2031 (1998); C.A. Sackett, J.M. Gerton, M. Welling, and R.G. Hulet, ibid. 82, 876 (1999).

[3] A. Gammal, L. Tomio, T. Frederico, and Ph. Chomaz, J. Phys. B 33, 4053 (2000).

[4] M. Desaix, D. Anderson, and M. Lisak, J. Opt. Soc. Am. B 8, 2082 (1991).

[5] A.L. Fetter, Phys. Rev. A 53, 4245 (1996).

[6] V.M. Perez-Garcia, H. Michinel, J.I. Cirac, M. Lewenstein, and P. Zoller, Phys. Rev. A 56, 1424 (1997).
[7] M. Houbiers and H.T.C. Stoof, Phys. Rev. A 54, 5055 (1996).

[8] T. Tsurumi and M. Wadati, J. Phys. Soc. Jpn. 68, 1531 (1999); T. Tsurumi, H. Morise, and M. Wadati, ibid. 68, 1871 (1999).

[9] P.A. Ruprecht, M.J. Holland, K. Burnett, and M. Edwards, Phys. Rev. A 51, 4704 (1995).

[10] A. Gammal, T. Frederico, and L. Tomio, Phys. Rev. E 60, 2421 (1999).

[11] V.E. Zakharov, Zh. Éksp. Teor. Fiz. 62, 1745 (1972) [Sov. Phys. JETP 35, 908 (1972)]; V.E. Zakharov and V.S. Synakh, ibid. 68, 940 (1975) [ibid. 41, 465 (1975)].

[12] L. Bergé, Phys. Rep. 303, 260 (1998).

[13] E.A. Kuznetsov, A.M. Rubenchik, and V.E. Zakharov, Phys. Rep. 142, 103 (1986). 
[14] M.I. Weinstein, Commun. Math. Phys. 87, 567 (1983).

[15] L. Bergé, Phys. Plasmas 4, 1227 (1997).

[16] C. Huepe, S. Métens, G. Dewel, P. Borckmans, and M.E. Brachet, Phys. Rev. Lett. 82, 1616 (1999).

[17] E. Tiesinga, A.J. Moerdijk, B.J. Verhaar, and H.T.C. Stoof, in Bose-Einstein Condensation, edited by A. Griffin, D.W. Snoke, and S. Stringari (Cambridge University Press, Combridge, 1995), p. 465; S. Inouye, M.R. Andrews, J. Stenger, H.J. Miesner, D.M. Stamper-Kurn, and W. Ketterle, Nature (London) 392, 151 (1998).

[18] Y.B. Gaididei, K.O. Rasmussen, and P.L. Christiansen, Phys. Rev. E 52, 2951 (1995); Yu.B. Gaididei, J. Schjodt-Eriksen, and P.L. Christiansen, ibid. 60, 4877 (1999).

[19] G. Fibich and G.C. Papanicolaou, SIAM (Soc. Ind. Appl. Math.) J. Appl. Math. 60, 183 (1999).

[20] N. Akhmediev, M.P. Das, and A.V. Vagov, in Condensed Matter Theories, edited by J.W. Clark and P.V. Panat (Nova, New York, 1997), Vol. 12, pp. 17-23, Int. J. Mod. Phys. B 13, 625 (1999).

[21] A. Gammal, T. Frederico, L. Tomio, and Ph. Chomaz, Phys. Rev. A 61, 051602(R) (2000).

[22] N.G. Vakhitov and A.A. Kolokolov, Izv. Vyssh. Uchebn. Zaved. Radiofiz. 16, 1020 (1973) [Radiophys. Quantum Electron. 16, 783 (1975)].
[23] I.V. Barashenkov, A.D. Gocheva, V.G. Makhankov, and I.V. Puzynin, Physica D 34, 240 (1988); I.V. Barashenkov and E.Yu. Panova, ibid. 69, 114 (1993).

[24] L. Bergé, T.J. Alexander, and Yu.S. Kivshar, Phys. Rev. A 62, 023607 (2000).

[25] L.D. Landau and E.M. Lifshitz, Mechanics (Pergamon, Oxford, 1976).

[26] G. Hechenblaikner, O.M. Marago, E. Hodby, J. Arlt, S. Hopkins, and C.J. Foot, Phys. Rev. Lett. 85, 692 (2000).

[27] D. Anderson, Phys. Rev. A 27, 3135 (1983).

[28] J.J.G. Ripoll and V.M. Perez-Garcia, Phys. Rev. A 59, 2220 (1999); J.J. Garcia-Ripoll, V.M. Perez-Garcia, and P. Torres, Phys. Rev. Lett. 83, 1715 (1999).

[29] S.N. Vlasov, V.A. Petrishchev, and V.I. Talanov, Izv. Vyssh. Uchebn. Zaved. Radiofiz. 14, 1353 (1971) [Sov. Radiophys. 14, 1062 (1974)].

[30] A. Gammal, T. Frederico, L. Tomio, and F.Kh. Abdullaev, Phys. Lett. A 267, 305 (2000).

[31] H.T.C. Stoof, J. Stat. Phys. 87, 1353 (1997).

[32] S. Stringari, Phys. Rev. Lett. 77, 2360 (1996); K.G. Singh and D.S. Rokhsar, ibid. 77, 1667 (1996); P.A. Ruprecht, M. Edwards, K. Burnett, and C.W. Clark, Phys. Rev. A 54, 4178 (1996).

[33] M.I. Weinstein, Commun. Pure Appl. Math. 39, 51 (1986). 